\title{
Assessing 'No Evidence of Disease Activity' Status in Patients with Relapsing-Remitting Multiple Sclerosis Receiving Fingolimod in Routine Clinical Practice: A Retrospective Analysis of the Multiple Sclerosis Clinical and Magnetic Resonance Imaging Outcomes in the USA (MS-MRIUS) Study
}

\author{
Bianca Weinstock-Guttman ${ }^{1} \cdot$ Jennie Medin $^{2} \cdot$ Nasreen Khan $^{3} \cdot$ Jonathan R. Korn $^{4}$. \\ Ellen Lathi ${ }^{5} \cdot$ Jason Silversteen $^{6} \cdot$ Jonathan Calkwood $^{7} \cdot$ Diego Silva $^{2} \cdot$ \\ Robert Zivadinov ${ }^{8,9} \cdot$ On behalf of the MS-MRIUS Study Group
}

Published online: 21 December 2017

(C) The Author(s) 2017. This article is an open access publication

\begin{abstract}
Background 'No evidence of disease activity' (NEDA), a composite measure of clinical and magnetic resonance imaging outcomes, provides a comprehensive assessment of disease activity, but is not extensively reported in clinical practice. NEDA-3 is defined as patients with no new/ enlarged $\mathrm{T} 2$ or gadolinium-enhancing lesions, no relapses, and no disability progression (according to Expanded
\end{abstract}

Electronic supplementary material The online version of this article (https://doi.org/10.1007/s40263-017-0482-4) contains supplementary material, which is available to authorized users.

Bianca Weinstock-Guttman

bw8@buffalo.edu

1 State University of New York at Buffalo, Jacobs Multiple Sclerosis Center for Treatment and Research, Jacobs Pediatric Multiple Sclerosis Center of Excellence, New York State Multiple Sclerosis Consortium, Buffalo, NY, USA

2 Novartis Pharma AG, Basel, Switzerland

3 IQVIA, Basel, Switzerland

4 IQVIA, Burlington, MA, USA

5 The Elliot Lewis Center for Multiple Sclerosis Care, Boston, MA, USA

6 Christiana Care Multiple Sclerosis Center, Newark, DE, USA

7 Minneapolis Clinic of Neurology, Golden Valley, MN, USA

8 Department of Neurology, Buffalo Neuroimaging Analysis Center, Jacobs School of Medicine and Biomedical Sciences, University at Buffalo, State University of New York at Buffalo, Buffalo, NY, USA

9 Translational Imaging Center at Clinical Translational Science Institute, University at Buffalo, State University of New York at Buffalo, Buffalo, NY, USA
Disability Status Scale scores). NEDA-4 comprises the components of NEDA-3 and a fourth criterion of $\leq 0.4 \%$ annualized brain volume loss.

Objective The objective of this study was to assess NEDA status among patients with relapsing-remitting multiple sclerosis receiving fingolimod in clinical practice.

Methods Clinical and magnetic resonance imaging data were retrospectively collected from 590 patients who initiated fingolimod at 33 multiple sclerosis centers in the USA. Patients were required to have a magnetic resonance imaging scan in the 6 months before or 1 month after fingolimod initiation (index period) and in the 9-24 months after fingolimod initiation (post-index period). Magnetic resonance imaging data were systematically quantified at a centralized reading facility. The proportions of patients with NEDA-3 or NEDA-4 status during fingolimod treatment were assessed.

Results During the follow-up period (median: 16 months), data to assess NEDA-3 and NEDA-4 were available for 586 and 325 patients, respectively. In the post-index period, $58.7 \%$ of patients achieved NEDA-3 status (no relapses, $85.2 \%$; no new/enlarged T2/gadolinium-enhancing lesions, 76.3\%; no disability progression, $87.9 \%$ ) and $37.2 \%$ achieved NEDA-4 status (no relapses, $86.5 \%$; no new/enlarged T2/gadolinium-enhancing lesions, $78.8 \%$; no disability progression, $91.1 \%$; brain volume loss $\leq 0.4$, $58.2 \%$ ).

Conclusion Among patients receiving fingolimod, over half achieved NEDA-3 status and over one-third achieved NEDA-4 status. 


\section{Key Points}

During 16 months of treatment with fingolimod, $59 \%$ of patients had no evidence of disease activity as defined by no relapses, no disability progression, and no active brain lesions on follow-up magnetic resonance imaging scans.

Approximately $37 \%$ of patients had no evidence of disease activity during fingolimod treatment when including the criterion of annualized brain volume loss $\leq 0.4 \%$ in the definition.

This study highlights the feasibility of using a systematic and consistent methodology in evaluating the proportion of patients who achieve no evidence of disease activity in routine clinical practice; individuals who achieve no evidence of disease activity status may have better long-term outcomes than those who do not.

\section{Introduction}

Multiple sclerosis (MS) is a chronic autoimmune disease of the central nervous system. Most patients present with relapsing-remitting MS, which is characterized by acute attacks of neurological dysfunction (relapses) followed by periods of complete or partial recovery [1]. Patients with MS require long-term treatment with disease-modifying therapies (DMTs) that can prevent relapses, slow disability, and target both the focal inflammatory and neurodegenerative aspects of MS to delay progression to irreversible disability [2]. Fingolimod (Gilenya ${ }^{\circledR}$; Novartis Pharma AG, Basel, Switzerland), a sphingosine-1 phosphate receptor modulator, was the first oral therapy to be approved for the treatment of patients with relapsing MS. In three pivotal, phase III randomized controlled trials (RCTs), fingolimod improved clinical and magnetic resonance imaging (MRI) outcomes vs. placebo or intramuscular interferon- $\beta-1$ a $\left(\right.$ Avonex $^{\circledR}$; Biogen Idec, Cambridge, Massachusetts, USA) [3-5].

Combined disease status assessments are increasingly being used to evaluate the overall impact of DMTs as a comprehensive alternative to measuring relapses, disability, and MRI outcomes in isolation [6]. Patients achieving freedom from clinical and MRI disease activity are said to have achieved 'no evidence of disease activity' (NEDA) and can be defined as those fulfilling the following three criteria: (1) no relapses, (2) no new/enlarged $\mathrm{T} 2$ or gadolinium-enhancing $\left(\mathrm{Gd}^{+}\right) \mathrm{T} 1$ lesions, and (3) no disability progression; this has been termed NEDA-3 [7, 8].
While NEDA-3 provides a stringent assessment of the initial focal inflammatory disease activity, the diffuse damage and neurodegenerative processes that characterize the complex underlying pathology of MS may be inadequately captured by these three criteria [6]. Therefore, an addition to NEDA-3 of a fourth criterion of no brain volume loss (BVL) has been suggested (NEDA-4) as an outcome that may provide a more comprehensive evaluation of disease activity $[6,8]$. Notably, in a recent post-hoc analysis of pooled data from placebo-controlled RCTs of fingolimod, patients receiving fingolimod were four times more likely to achieve NEDA-3 or NEDA-4 status than those receiving placebo [6].

Randomized controlled trials assess treatment efficacy in a carefully selected group of patients using a protocoldriven approach [9-11]. However, data generated according to rigorous experimental protocols in the selected patient population of RCTs may be difficult to extrapolate to patients in a real-world setting [9-11]. For example, unlike in clinical practice, outcomes in RCTs are assessed at pre-specified time points and patients are assessed in a standardized way for the duration of the trial. Furthermore, the frequency of MRIs conducted in RCTs is higher than in clinical practice. Robust real-world studies can, therefore, complement data from RCTs by investigating outcomes in a population of patients who are receiving treatment and being monitored according to routine clinical practice $[9,12,13]$.

There is a growing body of real-world data demonstrating the effectiveness of fingolimod on clinical and MRI outcomes [14]. The number of real-world studies assessing the effect of fingolimod on NEDA-3 is increasing, although to the best of our knowledge no studies report on NEDA-4 [14-16]. The challenges of assessing NEDA in clinical practice include the lack of standardization when collecting or quantifying MRI data, which can prevent direct comparisons between centers [17] and can lead to interrater variability when assessing lesions [18]. With regard to NEDA-4, BVL is not routinely evaluated in clinical practice and the MRI sequence most appropriate for assessing BVL, three-dimensional (3D) T1-weighted imaging, is not mandatory according to clinical guidelines $[17,19,20]$. These challenges limit the availability of MRI data for research purposes in clinical practice.

Of the real-world studies presenting data on NEDA-3 in patients receiving fingolimod, few have investigated cohorts identified from more than one center. In these studies, 35-59\% of patients achieved NEDA-3 status over 1-2 years of treatment [15, 16, 21, 22]. However, the methodology for collecting and analyzing MRI data was not always reported so it was not clear whether MRI outcomes were assessed using a standardized methodology [16]. Furthermore, few real-world studies have evaluated 
the impact of baseline disease activity on the proportion of patients able to achieve NEDA status during fingolimod treatment [23]. A need exists for robust real-world studies that generate high-quality clinical and MRI data, including for BVL, to assess the effectiveness of fingolimod with regard to patients achieving NEDA-3 and NEDA-4 status.

The Multiple Sclerosis Clinical and Magnetic Resonance Imaging Outcomes in the USA (MS-MRIUS) study was designed to investigate outcomes, including BVL, in patients receiving fingolimod in clinical practice [17, 24]. MS-MRIUS is novel in that it integrated MRI and clinical data recorded by physicians as part of routine clinical practice, and MRI outcomes collected from across participating centers were systematically quantified at the Buffalo Neuroimaging Analysis Center, a centralized reading facility.

In the present analysis using data from MS-MRIUS, we aimed to assess the proportions of patients achieving NEDA-3 and NEDA-4 status during treatment with fingolimod, and to evaluate the feasibility of measuring NEDA in clinical practice. The impact of baseline disease activity on the proportion of patients achieving NEDA status was also evaluated.

\section{Methods}

\subsection{Study Design}

MS-MRIUS was a large, multi-center, observational longitudinal cohort study that retrospectively analyzed patient data collected as part of routine clinical practice. Further details of the design and conduct of the study have been reported in Zivadinov et al. [17]. The study adhered to the Health Insurance Portability and Accountability Act and internal review board directives regarding patient privacy [17].

\subsection{Patient Selection}

All patients with relapsing-remitting MS in whom fingolimod treatment was initiated de novo were identified between August 2014 and June 2016 from 33 MS centers enrolled in MS-MRIUS across the USA. The date on which patients received their first dose of fingolimod was the index date. For study inclusion, patients were required to be aged 18-65 years at the index date, to have received fingolimod for at least 28 days, and to have available at least 12 months of pre-index and post-index medical chart data. Patients were also required to have an index MRI scan (within 6 months before and 1 month after the index date; the 'index period') and a post-index MRI scan (9-24 months after the index date) meeting the minimum quality criteria as detailed in Zivadinov et al. [17]. For the patient to be included, MRI scans were required to have been performed on 1.5 Tesla or 3 Tesla scanners and all scans were required to have a two-dimensional (2D) or 3D fluid attenuation inversion recovery sequence. For all eligible scans, 2D or 3D T1-weighted image sequences were collected.

Patients were excluded if they had participated in an interventional trial during the study period, had other neurological diseases affecting the central nervous system, had a history of alcohol/substance abuse, or had previously used fingolimod before initiating fingolimod in this study. Patients were also excluded if they had previously used natalizumab, owing to the fact that these patients often have highly active disease with a different disease profile from those who receive other DMTs (e.g., interferons/ glatiramer acetate). The exclusion of these patients thus ensured a more homogeneous patient cohort. Patients meeting the inclusion criteria and none of the exclusion criteria made up the intention-to-treat (ITT) cohort.

\subsection{Data Collection and Study Outcomes}

Clinical data, as recorded by the treating physician, were extracted from patient medical records and retrospectively analyzed. Medical records for all patients who met the eligibility criteria and had sufficient MRI data were included. Relapses documented in the patient medical records did not require definition by any pre-specified criteria. Disability was assessed according to changes in Expanded Disability Status Scale (EDSS) scores, which were estimated from neurological examination data in patient medical records. Disability progression was defined as an increase in the EDSS score between two time points of $\geq 1.5$ points if the baseline EDSS score was $0.0, \geq 1.0$ point if the baseline EDSS score was 1.5 , and $\geq 0.5$ points if the baseline EDSS score was > 5.0. Increases in EDSS scores were not required to be sustained for any period of time for patients to be classified as having disability progression. For patients with data for more than nine visits during the study period, information could be provided for one visit per 6 months; more extensive visits and visits closest to the scan dates were prioritized. Anonymized MRI and clinical data were integrated into a single dataset for analysis.

Magnetic resonance imaging scans were sent to the Buffalo Neuroimaging Analysis Center for a blinded systematic quantitative analysis of changes in brain volume and changes in the number of new/enlarged $\mathrm{T} 2$ and $\mathrm{Gd}^{+}$ lesions between the index and post-index periods. Brain volume loss was measured from images acquired at two different time points using Structural Image Evaluation, using Normalization, of Atrophy (SIENA) of scans 
obtained using 3D T1-weighted sequences when available, or via 2D T1-weighted sequences [17]. If multiple MRI scans were available, the index scan was the one closest to the index date and the post-index scan was the one furthest from the index date while patients were still persistent with fingolimod treatment in the post-index period.

Patients who achieved NEDA-3 or NEDA-4 status were defined as follows.

- NEDA-3: patients who had (1) no relapses, (2) no disability progression, and (3) no new/enlarged T2 or $\mathrm{Gd}^{+}$lesions.

- NEDA-4: patients who achieved the components of NEDA-3 in addition to the fourth criterion of no BVL (annualized BVL $\leq 0.4 \%$ ).

Patients for whom $\mathrm{Gd}^{+}$lesions were not assessed in the post-index period were assumed to have no $\mathrm{Gd}^{+}$lesions if they had no new/enlarged T2 lesions.

NEDA-3 and NEDA-4 were also assessed in patients who were stratified by the presence or absence of the following disease measures at baseline: one or more relapses in the 1-year pre-index period, disability progression between the pre-index and index period, or one or more $\mathrm{Gd}^{+}$lesions at the index MRI scan or active disease (defined as patients free from at least one of the following: one or more relapses in the 1-year pre-index period, disability progression between the pre-index and index period, or one or more $\mathrm{Gd}^{+}$lesions at the index MRI scan).

\subsection{Statistical Analyses}

For continuous variables, data are presented as the number of patients with valid/missing observations, mean, standard deviation, median, 95\% confidence interval, and minimum and maximum values. For categorical variables, data are presented as frequencies and related percentages per class level. $P$ values were calculated using the Pearson's chisquared test. Missing data were not imputed.

\section{Results}

\subsection{Study Population and Cohort Characteristics}

Of 601 patients with MRI data, 590 were eligible for inclusion in the ITT cohort. Reasons for exclusion are summarized in Fig. 1. At the index date, patients in the ITT cohort had a median age of 42.5 years, a median duration of MS of 8 years, and over three-quarters (78.6\%) were female. In the 1-year pre-index period, $23.1 \%$ of patients had received no DMTs and $70.5 \%$ had experienced no relapses. The proportion of patients with EDSS score progression between the pre-index to index period was
$18.5 \%$ and the proportion of patients with $\mathrm{Gd}^{+}$lesions at the index MRI scan was $21.5 \%$. Baseline demographic and disease characteristics are summarized in Table 1. Patients were followed for a median of 16 months during which time $85.8 \%$ of patients were persistent with fingolimod treatment. The largest proportion of patients received fingolimod for $>18$ to $\leq 24$ months (39.8\%; Table 2).

\subsection{Magnetic Resonance Imaging Sequences Available}

Brain volume loss could be evaluated only from MRI scans collected via 2D or 3D T1-weighted sequences using SIENA methodology. In the index period, 79.7 and $31.4 \%$ of patients had MRI scans collected using 2D and 3D T1weighted sequences, respectively; in the post-index period, the corresponding proportions of patients were 75.6 and $39.7 \%$, respectively. Conversely, almost all patients had MRI scans collected using fluid attenuation inversion recovery sequences: 99.5 and $99.3 \%$ in the index and postindex period, respectively (Table 3 ). T1 sequences with gadolinium contrast were collected for $91.9 \%$ of patients in the post-index period.

\subsection{Proportion of Patients Achieving NEDA-3 Status}

Of 586 patients for whom data were available to assess NEDA-3, 85.2\% had no relapses, $87.9 \%$ had no disability progression, and $76.3 \%$ had no new/enlarged $\mathrm{T} 2$ or $\mathrm{Gd}^{+}$ lesions in the post-index period. In total, $58.7 \%$ of patients ( $n=344 / 586)$ achieved NEDA-3 status in the post-index period (Fig. 2). Excluding $\mathrm{Gd}^{+}$lesions from the MRI lesion criterion of NEDA-3 did not affect the proportion of patients achieving NEDA-3 status [59.6\%; Fig. 1 of the Electronic Supplementary Material (ESM)].

When patients were stratified by baseline disease activity, a similar proportion achieved NEDA-3 status at follow-up irrespective of the absence or presence of relapses (59.6 vs. 56.6\%; Fig. 2a of the ESM) or disability progression (58.7 vs. $58.1 \%$; Fig. 2 b of the ESM). However, a higher proportion achieved NEDA-3 status if they had no $\mathrm{Gd}^{+}$lesions compared with those who had $\mathrm{Gd}^{+}$ lesions at baseline ( 61.8 vs. $47.5 \%$; $p=0.0047$; Fig. $2 \mathrm{c}$ of the ESM) or were free from active disease at baseline compared with those who had active disease (62.6 vs. $54.0 \%$; $p=0.0342$; Fig. $2 \mathrm{~d}$ of the ESM).

\subsection{Proportion of Patients Achieving NEDA-4 Status}

Of 325 patients for whom data were available to assess NEDA-4, 86.5\% had no relapses, $91.1 \%$ had no disability 
Fig. 1 Attrition of the study sample, by reason. ${ }^{\text {a}}$ To be eligible for analysis, magnetic resonance imaging (MRI) scans were required to have been performed on 1.5 Tesla or 3 Tesla scanners, within the allowed window, and to have quality information available. $B N A C$ Buffalo Neuroimaging Analysis Center, NEDA no evidence of disease activity

Table 1 Baseline demographic and disease characteristics of individuals in the intention-totreat (ITT) cohort

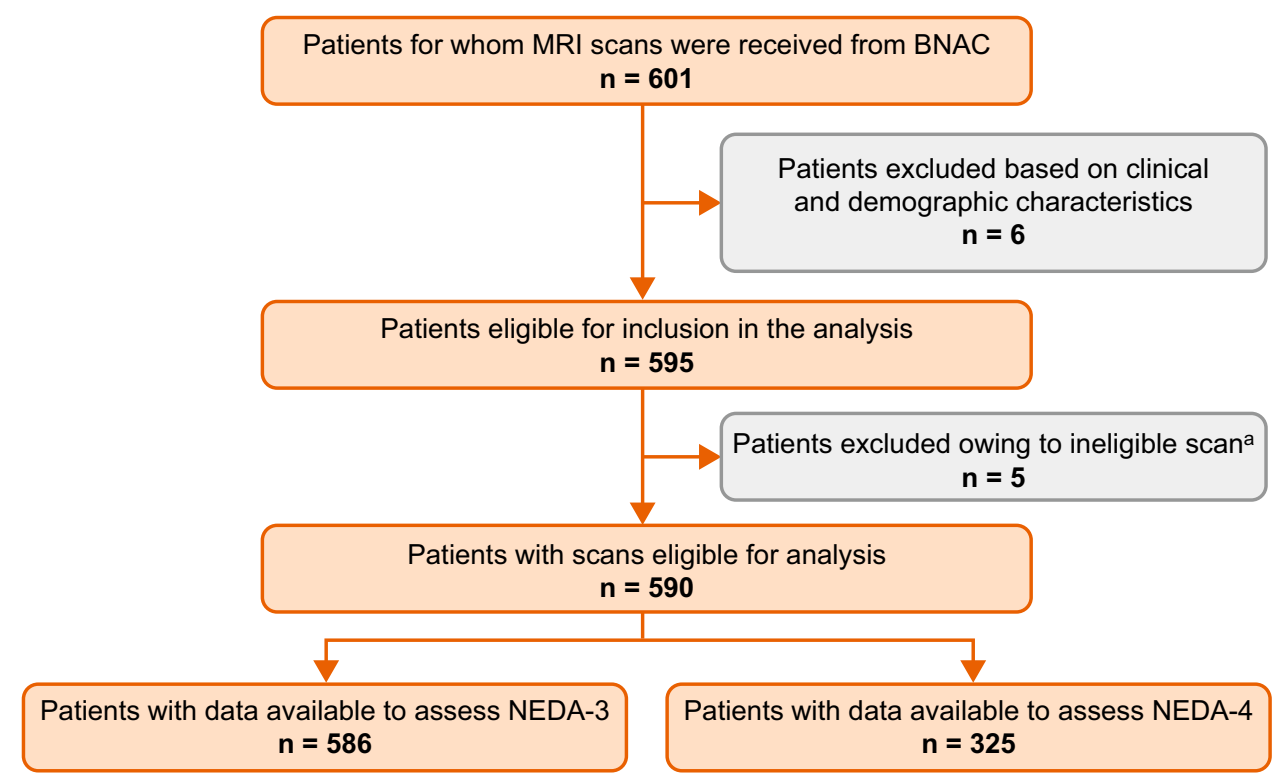

Characteristic

ITT cohort $(n=590)$

Age at index date, years

Mean (SD)

$42.5(9.8)$

Median (range)

$42.0(20-64)$

Female, $n(\%)$

464 (78.6)

Median duration of MS since diagnosis (range), years

$8.0(0-38)$

DMTs in the 1-year pre-index period, $n(\%)$

None

$136(23.1)$

Alemtuzumab

Dimethyl fumarate

$37(6.3)$

Glatiramer acetate

$158(26.8)$

$\operatorname{IFN} \beta-1 a^{a}$

$223(37.8)$

IFN $\beta-1 b^{b}$

68 (11.5)

$0(0.0)$

$5(0.8)$

Teriflunomide

$416 / 590(70.5)$

No relapses in the 1-year pre-index period, $n / N(\%)$

$43 / 232(18.5)$

$\mathrm{Gd}^{+}$lesions at index, $n / N(\%)$

$120 / 558(21.5)$

MRI findings at index date, mean (SD)

$\mathrm{T} 2$ lesion volume, $\mathrm{mL}(n=588)$

T1 lesion volume, $\mathrm{mL}(n=575)$

$\mathrm{Gd}^{+}$lesion number $(n=558)$

$\mathrm{Gd}^{+}$lesion volume, $\mathrm{mL}(n=558)$

Brain volume $^{\mathrm{c}}, \mathrm{mL}(n=539)$

$1527.2(97.6)$

$D M T$ disease-modifying therapy, $E D S S$ expanded disability status scale, $G d^{+}$gadolinium-enhancing, $I F N \beta$ interferon- $\beta, M R I$ magnetic resonance imaging, $M S$ multiple sclerosis, $S D$ standard deviation

${ }^{\text {a Avonex }}{ }^{\circledR}$, Rebif ${ }^{\circledR}$, or Plegridy ${ }^{\circledR}$

${ }^{\mathrm{b}}$ Betaseron ${ }^{\circledR}$ or Extavia ${ }^{\circledR}$

${ }^{\mathrm{c}}$ Brain parenchyma volume was measured by SIENAX (cross-sectional Structural Image Evaluation, using Normalization, of Atrophy) with scans obtained using three-dimensional T1-weighted images when available, or with two-dimensional T1-weighted images. Volumes were normalized by head size 
Table 2 Fingolimod treatment duration in the post-index period

\begin{tabular}{lc}
\hline Treatment duration & ITT cohort $(n=590)$ \\
\hline Treatment duration, months & \\
Mean, months (SD) & $18.1(7.2)$ \\
Median, months (range) & $18.4(1.6-54.1)$ \\
Treatment duration, $n(\%)$ & \\
$\leq 6$ months & $28(4.7)$ \\
$>6$ to $\leq 12$ months & $82(13.9)$ \\
$>12$ to $\leq 18$ months & $158(26.8)$ \\
$>18$ to $\leq 24$ months & $235(39.8)$ \\
$>24$ months & $87(14.7)$ \\
\hline
\end{tabular}

ITT intention-to-treat, $S D$ standard deviation

progression, $78.8 \%$ had no new/enlarged $\mathrm{T} 2$ or $\mathrm{Gd}^{+}$ lesions, and $58.2 \%$ had no BVL in the post-index period. In total, $37.2 \%$ of patients ( $n=121 / 325)$ achieved NEDA-4 status in the post-index period (Fig. 3). Excluding $\mathrm{Gd}^{+}$ lesions from the MRI lesion criterion of NEDA-4 did not affect the proportion of patients achieving NEDA-4 status (37.5\%; Fig. 3 of the ESM).
When patients were stratified by baseline disease activity, a similar proportion achieved NEDA-4 status at follow-up, irrespective of the absence or presence of relapses (39.3 vs. $31.9 \%$; Fig. 4a of the ESM). However, the proportion of patients who achieved NEDA-4 status was greater in those without vs. those with disability progression at baseline (38.8 vs. $14.3 \%$; $p=0.0245$; Fig. $4 \mathrm{~b}$ of the ESM), in those without vs. those with $\mathrm{Gd}^{+}$lesions at baseline ( 40.1 vs. $25.8 \% ; p=0.0376$; Fig. $4 \mathrm{c}$ of the ESM), and in those who were free from active disease vs. those who had active disease at baseline (42.4 vs. $29.9 \%$; $p=0.0212$; Fig. $4 \mathrm{~d}$ of the ESM).

\section{Discussion}

This study used quantitative MRI data collected from 33 centers and systematically analyzed them at a centralized reading facility alongside clinical data to evaluate the feasibility of assessing NEDA in patients receiving fingolimod in clinical practice. To the best of our knowledge, this is the first publication that includes BVL in the assessment of NEDA in patients receiving fingolimod in

Table 3 Availability of magnetic resonance imaging scans

\begin{tabular}{lcc}
\hline Characteristic & Index scan $(n=590)$ & Post-index scan $(n=590)$ \\
\hline Pulse sequence, $n(\%)$ & & $446(75.6)$ \\
2D T1 & $470(79.7)$ & $234(39.7)$ \\
3D T1 & $185(31.4)$ & $122(20.7)$ \\
2D T1 and 3D T1 & $98(16.6)$ & $586(99.3)$ \\
FLAIR & $587(99.5)$ & $542(91.9)$ \\
T1 sequence with gadolinium contrast, $n(\%)$ & $558(94.6)$ &
\end{tabular}

Brain parenchyma volume was measured by SIENAX (cross-sectional Structural Image Evaluation, using Normalization, of Atrophy) with scans obtained using three-dimensional T1-weighted images when available, or with two-dimensional T1-weighted images

$2 D$ two-dimensional, $3 D$ three-dimensional, FLAIR fluid-attenuated inversion recovery

Fig. 2 Proportion of patients achieving the individual components of no evidence of disease activity (NEDA)-3 and overall NEDA-3 status in the post-index period $(n=586)$. ${ }^{a}$ Patients for whom gadoliniumenhancing $\left(\mathrm{Gd}^{+}\right)$lesions were not assessed in the post-index period were assumed to have no $\mathrm{Gd}^{+}$lesions if they had no new/ enlarged T2 lesions

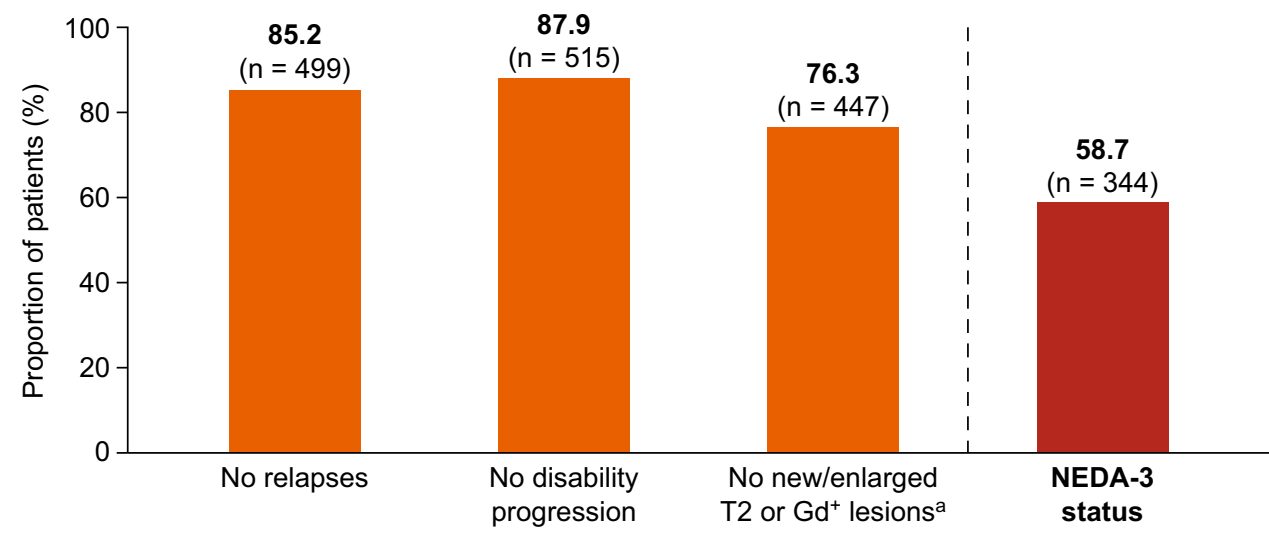


Fig. 3 Proportion of patients achieving the individual components of no evidence of disease activity (NEDA)-4 and overall NEDA-4 status in the post-index period $(n=325)$. ${ }^{\text {a }}$ Patients for whom gadoliniumenhancing $\left(\mathrm{Gd}^{+}\right)$lesions were not assessed in the post-index period were assumed to have no $\mathrm{Gd}^{+}$lesions if they had no new/ enlarged T2 lesions. $B V L$ brain volume loss

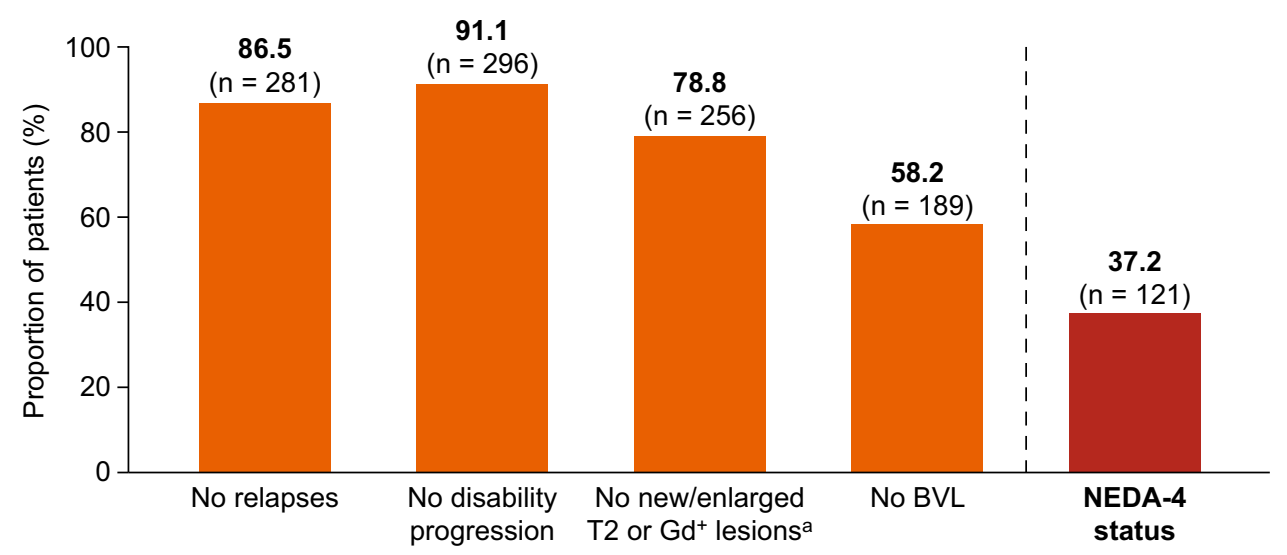

clinical practice. This study is important in that it provides insight into the proportions of patients achieving NEDA-3 and NEDA-4 status during fingolimod treatment in clinical practice, rather than in the experimental setting of an RCT.

In our study, higher proportions of patients achieved NEDA-3 (58.7\%) and NEDA-4 (37.2\%) status over 16 months than in a post-hoc analysis of RCT data from patients who received fingolimod treatment for 24 months (31.0 and 19.7\%, respectively) [6]. This was despite the fact that the criterion used for disability progression was more sensitive than that used in the post-hoc analysis of RCT data, in which disability progression was required to be confirmed after 6 months. Furthermore, the post-hoc analysis of RCT data did not include $\mathrm{Gd}^{+}$lesions in the criterion of active MRI lesions. However, excluding $\mathrm{Gd}^{+}$ from our analyses of NEDA did not impact on the proportion of patients achieving NEDA status. Indeed, when $\mathrm{Gd}^{+}$lesions were investigated in the post-hoc analysis of RCT data, nearly every $\mathrm{Gd}^{+}$lesion was found to be represented by a new/enlarged $\mathrm{T} 2$ lesion [6].

Possible reasons for the difference in results between our study and the study using pooled RCT data include the shorter follow-up period in the present analysis vs. that of Kappos et al. [6]; in support of this, a long-term real-world study demonstrated that patients lose NEDA status over time, and that this is particularly apparent in the first few years of follow-up [25]. In addition, $71 \%$ of patients in our study did not experience a relapse in the year before fingolimod initiation, whereas for inclusion in the fingolimod phase III RCTs assessed in the post-hoc analysis, patients were required to have at least one relapse in the previous year. The patients included in our analysis are therefore likely to have had lower baseline disease activity than the patients included in the Kappos et al. post-hoc analysis, which may have impacted on outcomes [3-5]. Differences in patient monitoring and quantification of clinical and MRI data between the present real-world study and the fingolimod phase III RCTs may also have accounted for the variation in results; this is particularly likely with regard to
MRI data, which was the major driver of NEDA-3 activity in the post-hoc analysis of RCT data [6]. For example, in fingolimod phase III RCTs, MRI scans were performed at 6- to 12-month intervals and captured the cumulative number and volume of lesions over the study period [3-6].

Before fingolimod initiation, less than half of patients experienced one or more of relapses in the year before the index, disability progression between the pre-index and index period, or $\mathrm{Gd}^{+}$lesions on the index MRI scan. Patients often switch DMTs owing to disease activity [8]; however, the reason for a treatment switch was unavailable from patients' medical records in the present study. Magnetic resonance imaging activity has been shown to be a strong predictor of switch behavior and may have caused the patient switch in our population [26]. However, longitudinal changes in MRI outcomes over time, including new/enlarged T2 lesions and BVL, could not be assessed at baseline owing to a lack of availability of pre-index MRI scans for the majority of patients in the ITT cohort [26]. Another possible explanation for low disease activity before fingolimod initiation may be that patients switched to fingolimod for reasons other than disease activity. Other common reasons for switching between DMTs include a doctor's recommendation, perceived treatment efficacy, adverse events and tolerability issues, and dislike of injections or infusions [27].

The presence or absence of baseline disease activity may affect whether patients achieve NEDA status during fingolimod treatment. In the present study, when patients were stratified by baseline disease activity, the presence of $\mathrm{Gd}^{+}$lesions and active disease significantly reduced the proportion of patients who achieved NEDA-3 and NEDA-4 status during fingolimod treatment. The presence of disability progression at baseline significantly reduced the proportion of patients who achieved NEDA-4, but not NEDA-3, status during fingolimod treatment. The baseline characteristics that affected NEDA-3 status in the present study differ from that of a single-center real-world study, which demonstrated that patients with more relapses or a 
higher EDSS score at baseline were at increased risk of not achieving NEDA-3 status 2 years after fingolimod initiation [23]. The difference between our study and that of Giuliani et al. may reflect differences in the patient populations being assessed and the criteria according to which stratification was performed. To the best of our knowledge, no real-world studies have assessed the impact of baseline disease activity on NEDA-4 status during fingolimod treatment.

NEDA-3 status is being increasingly assessed in clinical practice, including in studies of patients receiving fingolimod [15, 16, 21, 22, 28, 29]. In these real-world studies, the proportion of patients who achieved NEDA-3 status over 1-2 years was generally lower than that observed in our study [15, 16, 21, 22, 28, 29]. The largest of these studies presented data for a cohort of 662 patients from 11 Turkish centers who were regularly monitored for over 2 years, and reported that a lower proportion of patients (35\%) achieved NEDA-3 status than in the present study [16]. Variations in the patient populations being investigated, study design, and the duration of the followup period over which NEDA status was assessed may account for some of the differences in results between the present study and previous studies and prevent direct comparisons between studies [15, 16, 28, 29]. Differences in the NEDA criteria may also have accounted for some of the variation in results. However, a limitation of the Turkish study and other real-world studies is that definitions of the individual components of NEDA-3 were not always reported, and when definitions were provided there was often no consensus across the studies. Furthermore, in the majority of studies, it was unclear whether MRI data were collected and analyzed using a standardized approach [16].

There has been a shift in treatment goals towards managing patients with MS using DMTs that target both clinical and MRI measures of disease activity [7, 8, 30]. Patients achieving NEDA may have better long-term outcomes than those with clinical or subclinical breakthrough disease, highlighting the need for early and effective intervention [25, 30]. A longitudinal cohort study found that NEDA-3 status at 2 years could be used as a predictive measure of no disease progression at 7 years [25]. However, while NEDA-3 provides comprehensive insight into inflammatory activity in the brain, it does not account for diffuse central nervous system damage and neurodegeneration [6, 8, 31]. Treatment effect on BVL has been shown to correlate with disability progression, as well as having predictive value for future disability, demonstrating the importance of assessing BVL [8, 31-33]. The addition of BVL to NEDA-3 therefore increases the stringency of the assessment by providing a more comprehensive insight into the underlying pathology of MS without affecting its sensitivity to treatment effect $[6,8,31,34]$.

Measuring NEDA in clinical practice will necessitate the collection of high-quality MRI data using a systematic methodology [34]. Furthermore, BVL is not routinely collected in clinical practice owing to methodological and infrastructure constraints [34]. The MRI sequence most suitable for assessing BVL_-3D T1-weighted imaging-is not routinely conducted in clinical practice, and both Magnetic Resonance Imaging in MS (MAGNIMS) and Consortium of MS Centers (CMSC) consensus guidelines for the use of MRI in the diagnosis and follow-up of patients with MS recommend this protocol only as an optional sequence $[17,19,20,34]$. Therefore, in the absence of data on BVL, it will be necessary to identify a proxy measure of brain atrophy that can be readily evaluated from data collected during routine clinical practice to assess NEDA-4.

A strength of this study is that it combined data for a large number of patients across several MS centers in USA, supporting the feasibility of a systematic evaluation of patient data collected as part of large observational studies. Furthermore, the methodological requirements for image generation and the centralized facility used for image quantification ensured data quality. A limitation of this study is that data were extracted from medical records, which limited the availability of certain data, or the availability of data for some patients if these were not recorded consistently across MS centers. In particular, the reason for the treatment switch to fingolimod was not available from patient medical records, thus, it was not possible to ascertain whether patients were switching treatment owing to disease activity, tolerability issues, or for other reasons; this is a major limitation of retrospective analysis of patient medical records. Another limitation of the study is that changes in EDSS scores were not required to be sustained over any period of time for patients to be classified as having disability progression. Patients with a temporary increase in EDSS scores (e.g., following a relapse) may therefore have been inaccurately identified as having disability progression [35], which may have resulted in the proportion of patients achieving NEDA status being underestimated.

\section{Conclusion}

This retrospective multi-center analysis used robust clinical and MRI data from MS-MRIUS to demonstrate that over half of patients receiving fingolimod achieved NEDA-3 and over a third achieved NEDA-4 status. This study highlights the feasibility of evaluating NEDA-3 and NEDA-4 in clinical practice in a cohort of patients from several centers using robust MRI data generated and 
assessed using a systematic and consistent methodology. Future studies should evaluate the long-term prognosis of patients who achieve NEDA status compared with those who do not in clinical practice. Furthermore, as our understanding of MS evolves, additional criteria may be included in the concept of NEDA. These may include patient-reported outcomes, which can assess the functional domains of MS, and the timed 25-Foot Walk Test, a measure of disability more commonly assessed in clinical routines than EDSS scores [30].

Acknowledgements The authors take full responsibility for the content of the article. The authors thank Dr. Anne-Marie Couto and Helen Schofield of Oxford PharmaGenesis, Oxford, UK for medical writing support, editorial assistance, and the collation and incorporation of comments from all authors (funded by Novartis Pharma AG, Basel, Switzerland).

\section{Compliance with Ethical Standards}

Funding This study was funded by Novartis Pharma AG. Oxford PharmaGenesis received funding from Novartis Pharma AG for medical writing support. IQVIA received funding from Novartis Pharma AG. The open access fee was covered by Novartis Pharma AG

Conflict of interest Bianca Weinstock-Guttman has received honoraria as a speaker and consultant for Biogen, EMD Serono, Sanofi Genzyme, Novartis, Teva Pharmaceuticals, and Genentech. She has received research funds from Biogen, EMD Serono, Sanofi Genzyme, Novartis, and Teva Pharmaceuticals. Jennie Medin and Diego Silva are paid employees of Novartis Pharma AG, Basel, Switzerland. Nasreen Khan is a paid consultant for IQVIA, Basel, Switzerland. Jonathan R. Korn was a paid employee of IQVIA (previously QuintilesIMS), Burlington, MA, USA at the time of this study. Ellen Lathi has received honoraria as a speaker and consultant for Acorda, Biogen, Genzyme, Novartis, Teva Pharmaceuticals, and Genentech. Jason Silversteen has no conflicts of interest to report. Jonathan Calkwood has performed advisory, consultancy, and speaker activities for Acorda, Biogen, EMD Serono, Genzyme, Novartis, Roche, and Teva; and has received grant/research support from Biogen, Celgene, Genzyme, Novartis, and Roche. Robert Zivadinov has received personal compensation from EMD Serono, Celgene, Claret Medical, Novartis, and Sanofi Genzyme for speaking and consultancy. He has received financial support for research activities from Claret Medical, IMS Health, InteKrin-Coherus, Novartis, Sanofi Genzyme, and Teva Pharmaceuticals.

Informed consent For this type of study, formal consent is not required.

Open Access This article is distributed under the terms of the Creative Commons Attribution-NonCommercial 4.0 International License (http://creativecommons.org/licenses/by-nc/4.0/), which permits any noncommercial use, distribution, and reproduction in any medium, provided you give appropriate credit to the original author(s) and the source, provide a link to the Creative Commons license, and indicate if changes were made.

\section{References}

1. Compston A, Coles A. Multiple sclerosis. Lancet. 2002;359(9313):1221-31. https://doi.org/10.1016/S0140-6736(02)08 220-X.

2. Derwenskus J. Current disease-modifying treatment of multiple sclerosis. Mt Sinai J Med. 2011;78(2):161-75. https://doi.org/10. 1002/msj.20239.

3. Calabresi PA, Radue EW, Goodin D, Jeffery D, Rammohan KW, Reder AT, et al. Safety and efficacy of fingolimod in patients with relapsing-remitting multiple sclerosis (FREEDOMS II): a doubleblind, randomised, placebo-controlled, phase 3 trial. Lancet Neurol. 2014;13(6):545-56. https://doi.org/10.1016/S1474-4422 (14)70049-3.

4. Cohen JA, Barkhof F, Comi G, Hartung HP, Khatri BO, Montalban X, et al. Oral fingolimod or intramuscular interferon for relapsing multiple sclerosis. N Engl J Med. 2010;362(5):402-15. https://doi.org/10.1056/NEJMoa0907839.

5. Kappos L, Radue EW, O'Connor P, Polman C, Hohlfeld R, Calabresi $\mathrm{P}$, et al. A placebo-controlled trial of oral fingolimod in relapsing multiple sclerosis. $\mathrm{N}$ Engl $\mathrm{J}$ Med. 2010;362(5):387-401. https://doi.org/10.1056/NEJMoa0909494.

6. Kappos L, De Stefano N, Freedman MS, Cree BA, Radue EW, Sprenger $\mathrm{T}$, et al. Inclusion of brain volume loss in a revised measure of 'no evidence of disease activity' (NEDA-4) in relapsing-remitting multiple sclerosis. Mult Scler. 2016;22(10):1297-305. https://doi.org/10.1177/135245851561 6701.

7. Stangel M, Penner IK, Kallmann BA, Lukas C, Kieseier BC. Towards the implementation of 'no evidence of disease activity' in multiple sclerosis treatment: the multiple sclerosis decision model. Ther Adv Neurol Disord. 2015;8(1):3-13. https://doi.org/ $10.1177 / 1756285614560733$.

8. Ziemssen T, Derfuss T, de Stefano N, Giovannoni G, Palavra F, Tomic D, et al. Optimizing treatment success in multiple sclerosis. J Neurol. 2016;263(6):1053-65. https://doi.org/10.1007/ s00415-015-7986-y.

9. Industry. TAotBP. Guidance: demonstrating value with real world data: a practical guide. http://www.abpi.org.uk/our-work/ library/guidelines/Documents/2011-06-13\%20ABPI\%

20 guidance $\% 20-\% 20$ Demonstrating $\% 20$ value $\% 20$ with $\% 20$ real $\%$ 20world\%20data.pdf. Accessed 11 May 2017.

10. Ziemssen T, Hillert J, Butzkueven H. The importance of collecting structured clinical information on multiple sclerosis. BMC Med. 2016;14:81. https://doi.org/10.1186/s12916-016-0627-1.

11. Trojano M, Tintore M, Montalban X, Hillert J, Kalincik T, Iaffaldano $\mathrm{P}$, et al. Treatment decisions in multiple sclerosis: insights from real-world observational studies. Nat Rev Neurol. 2017;13(2):105-18. https://doi.org/10.1038/nrneurol.2016.188.

12. Capkun G, Dahlke F, Lahoz R, Nordstrom B, Tilson HH, Cutter $\mathrm{G}$, et al. Mortality and comorbidities in patients with multiple sclerosis compared with a population without multiple sclerosis: an observational study using the US Department of Defense administrative claims database. Mult Scler Relat Disord. 2015;4(6):546-54. https://doi.org/10.1016/j.msard.2015.08.005.

13. Eraksoy M, Butzkueven H, Ziemssen T, Zivadinov R. Time for change: evolution of real-world evidence outcome measures in multiple sclerosis exemplified by fingolimod. Eur Neurol Rev. 2014;9(2):136-42.

14. Ziemssen T, Medin J, Couto A, Mitchell C. Multiple sclerosis in the real world: a systematic review of fingolimod as a case study. Autoimmun Rev. 2017;16(4):355-76.

15. Baroncini D, Ghezzi A, Annovazzi PO, Colombo B, Martinelli V, Minonzio G, et al. Natalizumab versus fingolimod in patients with relapsing-remitting multiple sclerosis non-responding to 
first-line injectable therapies. Mult Scler. 2016;22(10):1315-26. https://doi.org/10.1177/1352458516650736.

16. Terzi M, Kürtüncü M, Eraksoy M, Karabudak R, Tuncer A, Altunrende B, et al. Efficacy and the safety profile of fingolimod treatment in multiple sclerosis patients: real-life data from Turkey [poster]. Presented at the ECTRIMS Forum, 14-17 Sep 2016; London.

17. Zivadinov R, Khan N, Medin J, Christoffersen P, Price J, Korn JR, et al. An observational study to assess brain MRI change and disease progression in multiple sclerosis clinical practice: the MS-MRIUS study. J Neuroimaging. 2016;27(3):339-47. https:// doi.org/10.1111/jon.12411.

18. Erbayat Altay E, Fisher E, Jones SE, Hara-Cleaver C, Lee JC, Rudick RA. Reliability of classifying multiple sclerosis disease activity using magnetic resonance imaging in a multiple sclerosis clinic. JAMA Neurol. 2013;70(3):338-44. https://doi.org/10. 1001/2013.jamaneurol.211.

19. Rovira A, Wattjes MP, Tintore M, Tur C, Yousry TA, Sormani MP, et al. Evidence-based guidelines: MAGNIMS consensus guidelines on the use of MRI in multiple sclerosis: clinical implementation in the diagnostic process. Nat Rev Neurol. 2015;11(8):471-82. https://doi.org/10.1038/nrneurol.2015.106.

20. Traboulsee A, Simon JH, Stone L, Fisher E, Jones DE, Malhotra A, et al. Revised recommendations of the Consortium of MS Centers Task Force for a standardized MRI protocol and clinical guidelines for the diagnosis and follow-up of multiple sclerosis. AJNR Am J Neuroradiol. 2016;37(3):394-401. https://doi.org/10. 3174/ajnr.A4539.

21. Totaro R, Di Carmine C, Costantino G, Fantozzi R, Bellantonio $\mathrm{P}$, Fuiani A, et al. Fingolimod treatment in relapsing-remitting multiple sclerosis patients: a prospective observational multicenter postmarketing study. Mult Scler Int. 2015;2015:763418. https://doi.org/10.1155/2015/763418.

22. Totaro R, Costantino G, Bellantonia P, Danni M, Di Carmine C, Fantozzi R, et al. Efficacy of natalizumab and fingolimod in relapsing-remitting multiple sclerosis in real-world clinical setting. J Neurol Neurophysiol. 2016;6(6):1-6.

23. Giuliani M, Logoteta A, Prosperini L, Hirsch MN, Pozzilli C. Baseline characteristics associated with NEDA-3 status in fingolimod-treated patients with relapsing-remitting multiple sclerosis. Mult Scler Demyelinat Disord. 2017;2(1):10. https://doi. org/10.1186/s40893-017-0026-2.

24. Zivadinov R, Khan N, Medin J, Korn J, Bergsland N, Christoffersen $\mathrm{P}$, et al. Impact of fingolimod on MRI brain measures in routine clinical practice: interim results from a longitudinal observational, multicenter real-world outcome study in multiple sclerosis patients [poster]. Presented at the 32nd ECTRIMS Forum; 14-17 Sep 2016; London.
25. Rotstein DL, Healy BC, Malik MT, Chitnis T, Weiner HL. Evaluation of no evidence of disease activity in a 7-year longitudinal multiple sclerosis cohort. JAMA Neurol. 2015;72(2):152-8. https://doi.org/10.1001/jamaneurol.2014. 3537.

26. Teter B, Agashivala N, Kavak K, Chouhfeh L, Hashmonay R, Weinstock-Guttman B. Characteristics influencing therapy switch behavior after suboptimal response to first-line treatment in patients with multiple sclerosis. Mult Scler. 2014;20(7):830-6. https://doi.org/10.1177/1352458513513058.

27. Salter AR, Marrie RA, Agashivala N, Belletti DA, Kim E, Cutter GR, et al. Patient perspectives on switching disease-modifying therapies in the NARCOMS registry. Patient Prefer Adher. 2014;8:971-9. https://doi.org/10.2147/PPA.S49903.

28. Rasenack M, Rychen J, Andelova M, Naegelin Y, Stippich C, Kappos L, et al. Efficacy and safety of fingolimod in an unselected patient population. PLoS One. 2016;11(1):e0146190. https://doi.org/10.1371/journal.pone.0146190.

29. Yamout BI, Zeineddine MM, Tamim H, Khoury SJ. Safety and efficacy of fingolimod in clinical practice: the experience of an academic center in the Middle East. $J$ Neuroimmunol. 2015;289:93-7. https://doi.org/10.1016/j.jneuroim.2015.10.015.

30. Giovannoni G, Turner B, Gnanapavan S, Offiah C, Schmierer K, Marta M. Is it time to target no evident disease activity (NEDA) in multiple sclerosis? Mult Scler Relat Disord. 2015;4(4):329-33. https://doi.org/10.1016/j.msard.2015.04.006.

31. Giovannoni G, Tomic D, Bright JR, Havrdova E. "No evident disease activity": the use of combined assessments in the management of patients with multiple sclerosis. Mult Scler J. 2017;23(9):1179-87. https://doi.org/10.1177/1352458517703 193.

32. Bermel RA, Bakshi R. The measurement and clinical relevance of brain atrophy in multiple sclerosis. Lancet Neurol. 2006;5(2):158-70. https://doi.org/10.1016/S1474-4422(06)7034 9-0.

33. Popescu V, Agosta F, Hulst HE, Sluimer IC, Knol DL, Sormani MP, et al. Brain atrophy and lesion load predict long term disability in multiple sclerosis. J Neurol Neurosurg Psychiatry. 2013;84(10):1082-91. https://doi.org/10.1136/jnnp-2012-304094.

34. Zivadinov R, Jakimovski D, Gandhi S, Ahmed R, Dwyer MG, Horakova D, et al. Clinical relevance of brain atrophy assessment in multiple sclerosis: implications for its use in a clinical routine. Expert Rev Neurother. 2016;16(7):777-93. https://doi.org/10. 1080/14737175.2016.1181543.

35. Lublin FD, Baier M, Cutter G. Effect of relapses on development of residual deficit in multiple sclerosis. Neurology. 2003;61(11):1528-32. 